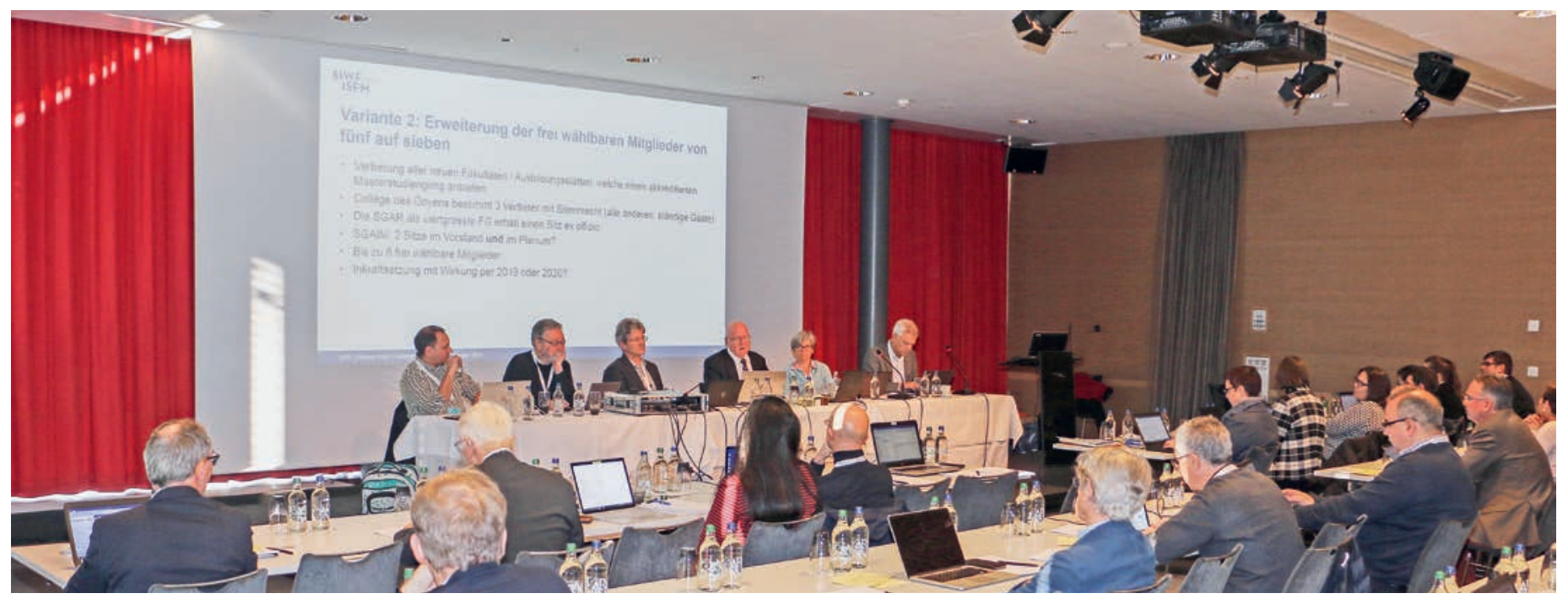

L'assemblée plénière de I'ISFM offre l'opportunité d'un échange direct entre la direction et les délégués.

\title{
Pour que la formation médicale reste en main des médecins
}

\section{Bruno Kesseli}

Dr méd. et lic. phil., rédacteur en chef

L'assemblée plénière 2018 de l'Institut suisse pour la formation médicale postgraduée et continue (ISFM) a, encore une fois, proposé un programme bien rempli tout en faisant une large place aux possibilités de discussion et d'intervention des participants. Le document stratégique qui comprend trois objectifs majeurs, dont le renforcement de la position de l'ISFM en tant qu'institution nationale du corps médical pour la formation médicale postgraduée et continue, a été adopté à l'unanimité.

Après avoir résumé, en introduction, la fonction de l'assemblée plénière, le président de l'ISFM, Werner Bauer, a rapidement donné la parole au tant attendu «Osler» [1], qui n’a plus besoin d'êtreprésenté. Le duo Osler-Bauer a rappelé ce que doit être un hôpital: $a$ place where the best that is known is taught to a group of the best students, en ajoutant, dans l'esprit d'un ceterum censeo, le constat suivant: the work of an institution, in which there is no teaching, is rarely first class.

\section{Flash d'information abondant}

Une fois ces principes établis, l'attention s'est portée sur une sélection de thèmes concrets et importants pour l'ISFM. Werner Bauer a présenté un premier aperçu de l'enquête annuelle conjointe de l'EPFZ et de l'ISFM et plus particulièrement le module destiné aux responsables des établissements de formation postgraduée portant sur la médecine personnalisée et sur le soutien de la formation postgraduée de la part de la direction hospitalière. L'évaluation a révélé que la créa- 


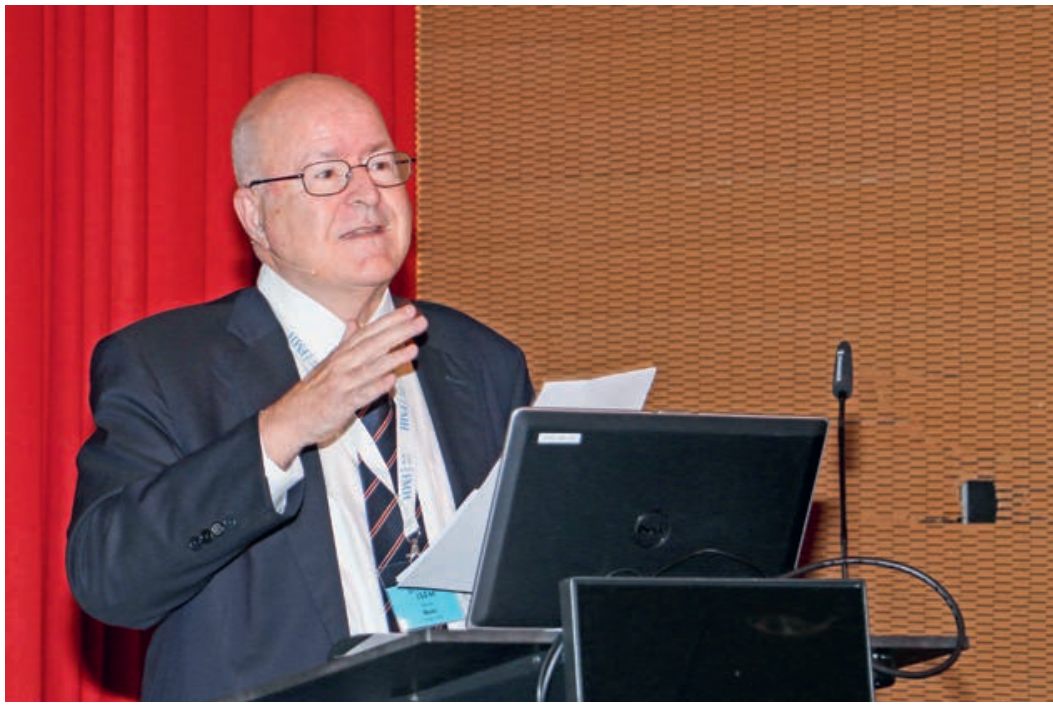

Werner Bauer, président de I'ISFM, a passé en revue les nombreux points de I'ordre du jour et présenté entre autres la stratégie de I'ISFM.

tion d'une nouvelle profession non médicale de Genetic Counselor a été rejetée à une large majorité. La tâche du Genetic Counselor aurait consisté à conseiller, sur mandat médical, les patients sur des questions génétiques concrètes. Par ailleurs, les personnes interrogées dans tous les domaines spécialisés estiment que les directeurs hospitaliers accordent une grande importance à la formation postgraduée. Et Werner Bauer de commenter que ce genre de déclarations sont légion.

Les autres informations concernaient les ateliers Teach the Teachers, organisés depuis quelques années en collaboration avec le Royal College of Physicians

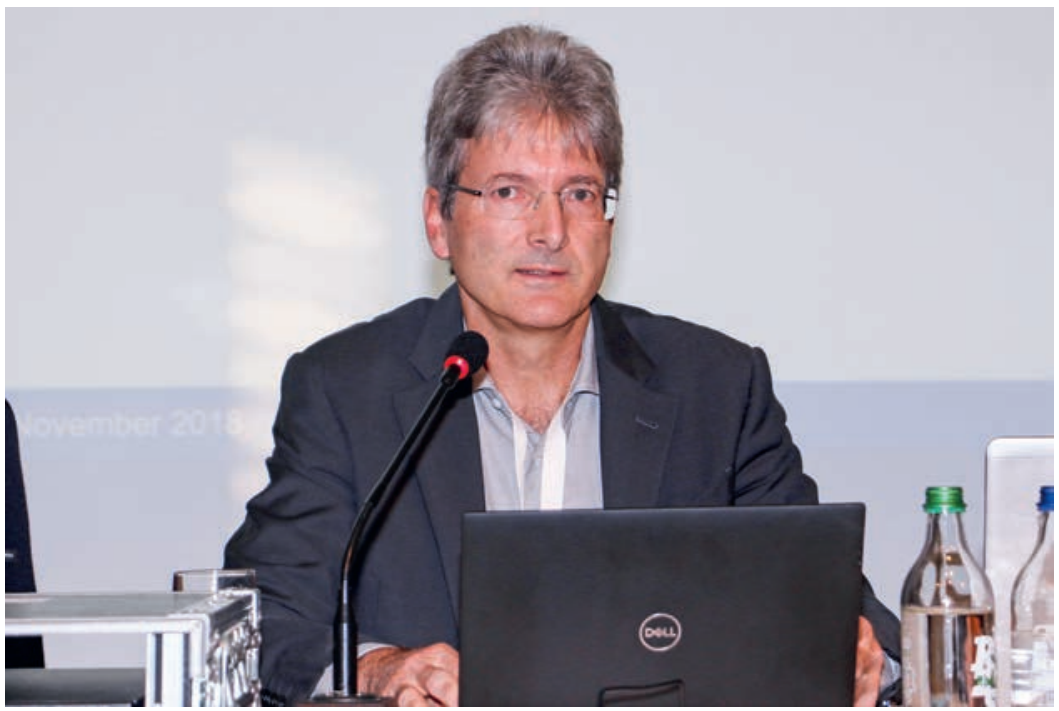

Le directeur de I'ISFM, Christoph Hänggeli, a pronostiqué un bilan équilibré lors de la présentation du budget 2019.
(RCP), et le symposium MedEd. Ces deux manifestations connaissent un vif succès. Les cours Teach the Teachers seront proposés en allemand et en français dès 2020. A cette fin, un pool de responsables de cours et d'instructeurs sera préalablement constitué avec le soutien du RCP. Toute personne intéressée peut se mettre directement en contact avec Werner Bauer.

Christoph Hänggeli, directeur de l'ISFM, a informé du remaniement du site Internet de l'ISFM. Dans les en quêtes clients, celui-ci est souvent critiqué pour son manque de clarté, ce qui en faisait un sujet de préoccupation. Le site devrait faire peau neuve en 2019. Christoph Hänggeli est ensuite passé à la loi sur les professions médicales (LPMéd), dont la révision cible deux objectifs principaux: un registre des médecins complet et fiable et la garantie des compétences linguistiques nécessaires à l'exercice de la profession. Ces deux objectifs sont réalisés depuis le $1^{\text {er }}$ janvier 2018. Divers acteurs - dont l'ASMAC, la FMH et l'ISFM - déplorent que les compétences en langues étrangères acquises avec la maturité ne soient pas prises en compte dans l'évaluation des compétences linguistiques. Des efforts sont actuellement déployés également sur le plan politique pour remédier à cette situation afin d'encourager les échanges de médecins entre les régions linguistiques. Christoph Hänggeli a par ailleurs abordé l'arrêt du Tribunal administratif fédéral de décembre 2017. Le tribunal avait dérogé à sa pratique habituelle en décidant que les formations approfondies et les attestations de formation complémentaire, dont la réglementation relève du droit privé et non de la LPMéd, tombaient dans le domaine public, ce qui permettait de recourir contre les décisions concernant les formations approfondies et les attestations de formation complémentaire. Estimant que cette pratique ne ferait que gonfler l'administration, l'ISFM a déposé un recours auprès du Tribunal fédéral.

\section{Budget 2019 et stratégie de l'ISFM}

Pour Christoph Hänggeli, la situation financière de l'ISFM permet de parler d'une tendance fondamentalement positive. Même s'il est difficile de prédire l'évolution des titres de spécialiste, un bilan équilibré a été budgété pour 2019

Selon Werner Bauer, la stratégie de l'ISFM se fonde sur la volonté de maintenir la formation médicale postgraduée et continue entre les mains des médecins. Les principaux objectifs, légèrement adaptés, sont les suivants:

- l'ISFM renforce sa position en tant qu'institution nationale du corps médical pour la formation médicale postgraduée et continue; 


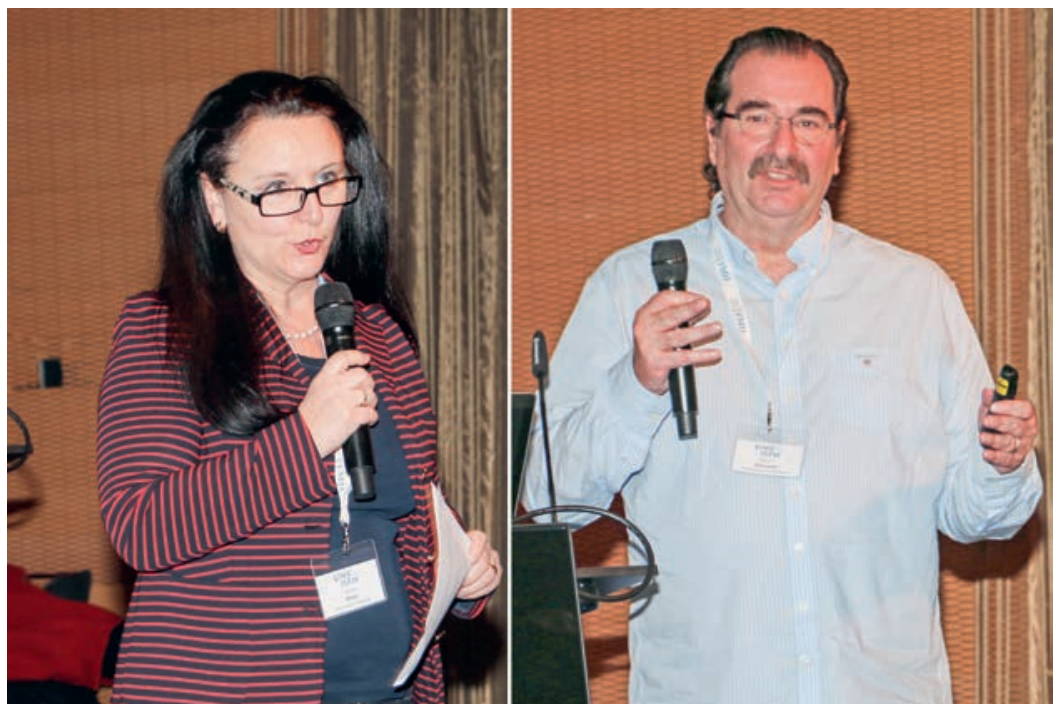

Les nouveaux élus au Comité de I'ISFM: Daniela Wiest et Marco Zalunardo.

- l'ISFM anticipe les évolutions dans le domaine de la formation postgraduée, élabore des mesures proactives et les applique;

- l'ISFM conçoit une formation continue et une évolution professionnelle tournées vers l'avenir (continuing professional development).

Werner Bauer souligne l'importance d'un positionnement actif de l'ISFM vis-à-vis de la politique, de l'administration et des organisations partenaires, mais aussi au sein du corps médical. Le document stratégique présenté et les explications du président ont manifestement convaincu les délégués, qui ont adopté la stratégie à l'unanimité.

\section{Composition du Comité et élections}

Après une brève introduction de Werner Bauer, la discussion a porté sur la composition du Comité de l'ISFM. Le

\section{Assemblée plénière de I'ISFM}

Organe autonome de la FMH, I'Institut suisse pour la formation médicale postgraduée et continue (ISFM) réunit tous les principaux acteurs et organismes du domaine de la formation médicale postgraduée et continue, et garantit aux médecins une formation de qualité élevée dans plus de 120 domaines spécialisés. L'assemblée plénière annuelle de I'ISFM remplit différentes fonctions. D'une part, les membres du plénum forment un Comité doté d'un pouvoir de décision et d'élection, qui traite les affaires relevant de son domaine de compétences à l'instar de la Chambre médicale de la FMH. De l'autre, I'assemblée, à laquelle sont également conviés des invités de provenances diverses, constitue une plate-forme chargée de transmettre des informations sur des thèmes spécifiques, de garantir l'échange d'expériences entre les participants et de débattre de toutes sortes de sujets. plénum a décidé de maintenir le nombre des membres à 19 et il a adopté une modification de la composition du Comité (avec deux abstentions et aucune opposition). Dès 2019, le Comité sera composé comme suit:

- représentation de toutes les facultés / tous les établissements de formation proposant une formation Master accréditée. Le Collège des Doyens désigne trois représentants avec droit de vote. Les autres comme l'OFSP, la CDS, $\mathrm{H}+$, la Commission des professions médicales, l'Institut d'enseignement médical (IML) et l'association Médecine universitaire suisse, sont des hôtes permanents;

- la Société suisse d'anesthésiologie et de réanimation (SSAR), en tant que quatrième plus grande société de discipline, se voit attribuer un siège "ex officio» comme les sociétés suisses de chirurgie (SSC), de pédiatrie (SSP), de psychiatrie et psychothérapie (SSPP) et de gynécologie et obstétrique (SSGO);

- la Société suisse de médecine interne générale (SSMIG) conserve deux sièges au Comité et au plénum; - un maximum de six membres au choix est éligible. La docteure Daniela Wiest de la Société suisse de neurologie a été nouvellement élue au Comité. Le professeur Marco Zalunardo a succédé "ex officio" au professeur Tiziano Cassina de la SSAR. Les autres membres du Comité dont le mandat de quatre ans était arrivé à échéance ont tous été réélus à l'unanimité, comme le vice-président Jean Pierre Keller.

La succession de Werner Bauer a également été abordée. Le président de l'ISFM n'étant pas à disposition pour un nouveau mandat, la Chambre médicale devra élire son successeur en mai 2020. Au cours de l'été 2019, le Comité de l'ISFM constituera une commission de prospection et publiera l'offre d'emploi.

\section{Logbook électronique en bonne voie}

Ces dernières années, le logbook électronique s'est assuré une place de choix à l'ordre du jour de l'assemblée plénière de l'ISFM. Après une adaptation de la roadmap, le projet est désormais en bonne voie, comme le constate Lukas Wyss. Cet informaticien médical qui travaille à l'ISFM comme chef de produit a donné à l'auditoire un tour d'horizon complet sur l'état des travaux et les étapes prévues.

Parmi les objectifs poursuivis, on peut citer le travail électronique sans rupture de médias (et sans papier), une navigation simplifiée, la standardisation des concepts nouveaux ou déjà utilisés (p. ex. les EPA*) et l'uniformisation des objectifs de formation sans modification des programmes de formation postgraduée. Le projet accorde une place très importante à la sécurité et la protection des données. 


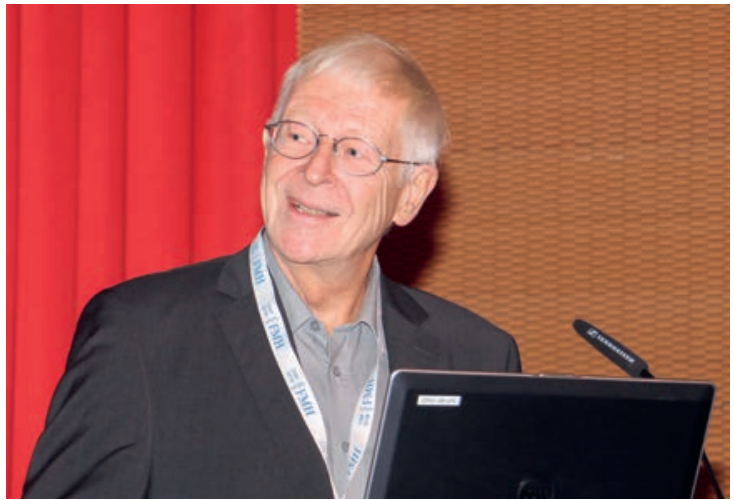

Compétent (également) en matière de radioprotection: Hans Rudolf Koelz.

\section{Révision des programmes de formation postgraduée}

Les délégués se sont prononcés sur des propositions de révision des programmes de formation postgraduée déposées par deux sociétés de discipline médicale. La Société suisse d'hématologie a proposé d'introduire dès 2019 l'examen de l'European Board comme épreuve écrite de l'examen de spécialiste en hématologie. Il s'agit ici d'un examen à choix multiples comportant 100 questions. La Société suisse de pédiatrie a proposé, quant à elle, de modifier les conditions pour les médecins formateurs, la réglementation actuelle ne répondant plus aux nouvelles évolutions. Elle demande «au moins deux ans d'activité dans un cabinet de pédiatre» au lieu du critère de deux ans d'activité dans un cabinet sous responsabilité propre. Les deux propositions de révision ont été adoptées par les délégués.

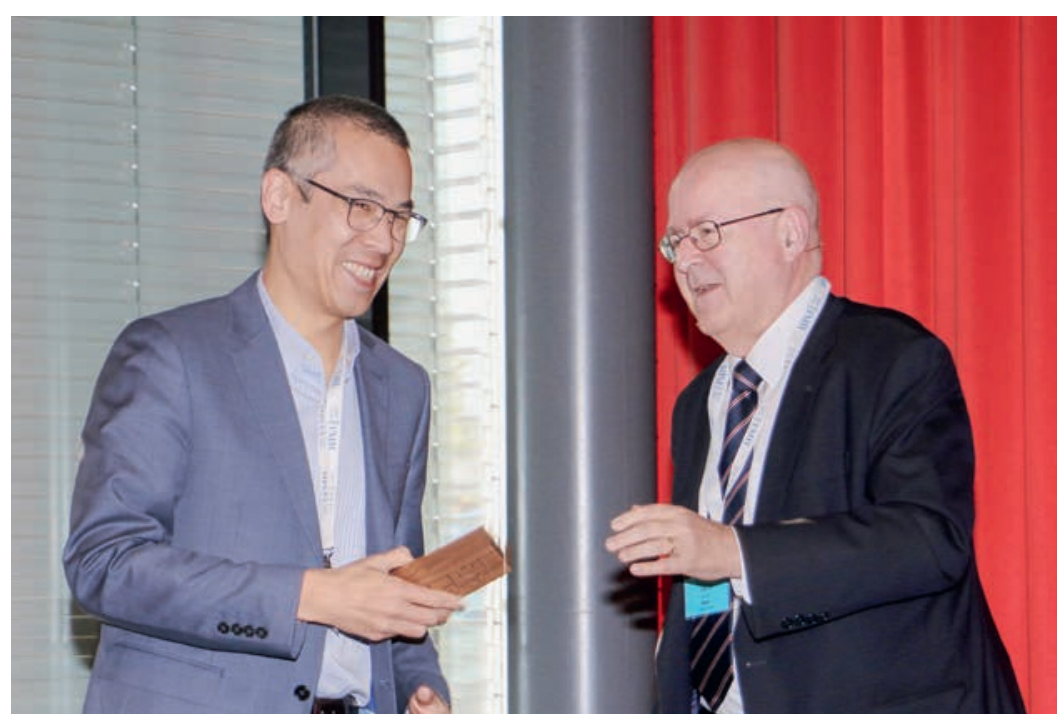

Werner Bauer remercie Ryan Tandjung (à gauche) pour ses longues années de collaboration en diverses fonctions.

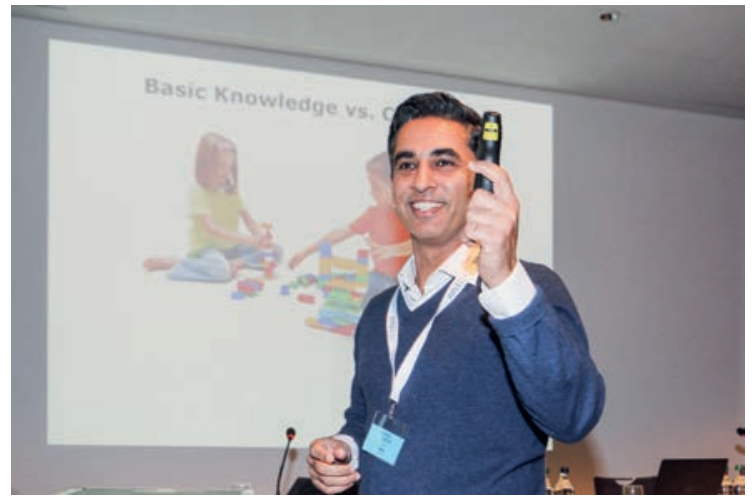

Manu Kapur a présenté la méthode de "l'échec productif».

\section{Radioprotection et un "au revoir»}

La radioprotection, évoquée par le professeur Hans $R u$ dolf Koelz, a sollicité pas mal de temps. La révision de l'ordonnance sur la radioprotection implique l'adaptation des programmes de formation postgraduée en radiologie, en médecine nucléaire et en radiooncologie. Cela vaut également pour certains autres programmes de formation postgraduée et de formation complémentaire. De plus, cette révision impose la création de nouvelles attestations de formation complémentaire. Les sociétés de discipline médicale concernées en ont déjà été informées. Après une longue séance de questions, les délégués ont approuvé à l'unanimité la procédure prévoyant la création de nouvelles attestations de formation complémentaire et leur adoption définitive par le Comité en mars 2019.

La matinée s'est achevée sur les adieux du Dr Ryan Tandjung, qui a été en contact régulier dans différentes fonctions avec l'ISFM pendant de nombreuses années. Dernièrement, il est intervenu activement au sein du Comité en tant que représentant de l'Office fédéral de la santé publique (OFSP), un mandat auquel il renonce désormais étant appelé à de nouvelles fonctions au sein de l'OFSP. Werner Bauer le remercie pour son engagement et lui adresse ses meilleurs vœux pour la suite de sa carrière.

\section{«Echec productif»}

L'après-midi a débuté sur un sujet passionnant grâce à l'intervention du professeur Manu Kapur. Cet ingénieur de Singapour occupe une chaire en Learning Sciences and Higher Education à l'EPFZ. Cet ancien footballeur professionnel de 43 ans, qui a dû mettre un terme à sa carrière suite à une blessure, s'est fait connaître grâce à sa méthode pédagogique de «l'échec productif». Cette méthode qu'il a présentée au plénum 


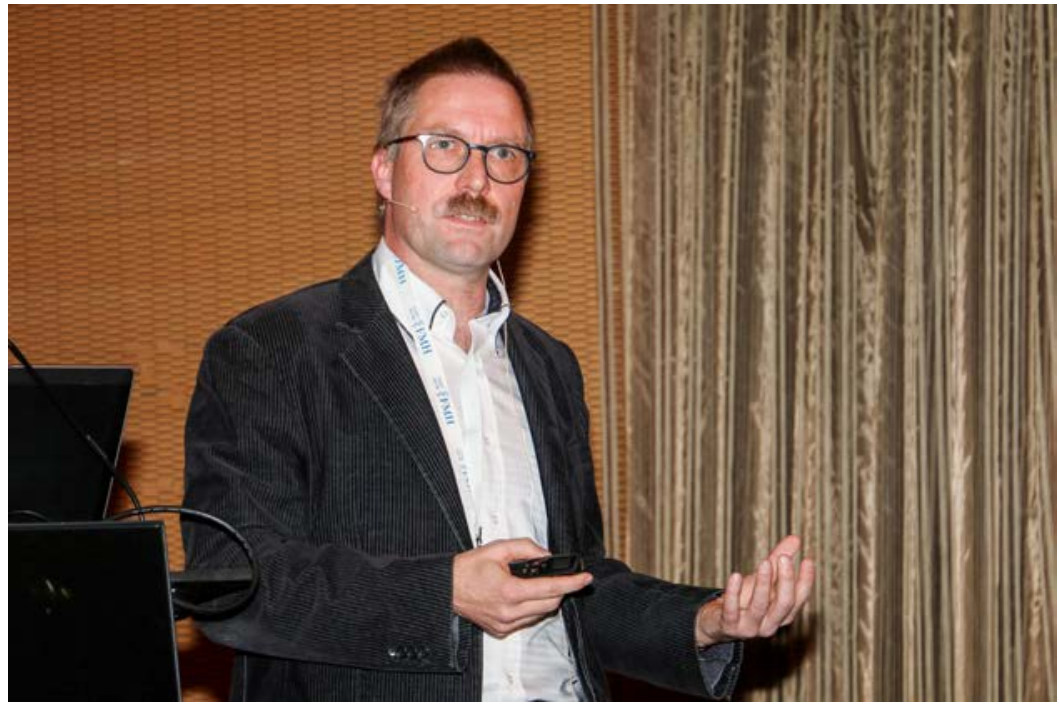

Jörg Goldhahn a expliqué l'engagement de I'EPFZ dans le domaine de la formation médicale postgraduée.

ouvrirait, selon lui, des perspectives intéressantes aussi pour la formation médicale. Une intégration de ses méthodes aux études de médecine ou dans la formation postgraduée et continue signifierait de recueillir des expériences pratiques dans certains domaines avant de les aborder de manière théorique. Manu Kapur accorde une place de choix à ce qu'on appelle le contextualized learning, qu'il oppose à l'apprentissage fragmenté ou décontextualisé qui est fréquemment utilisé aujourd'hui.

Comme l'a souligné le professeur Jörg Goldhahn dans l'exposé qui a suivi, les méthodes esquissées par Manu

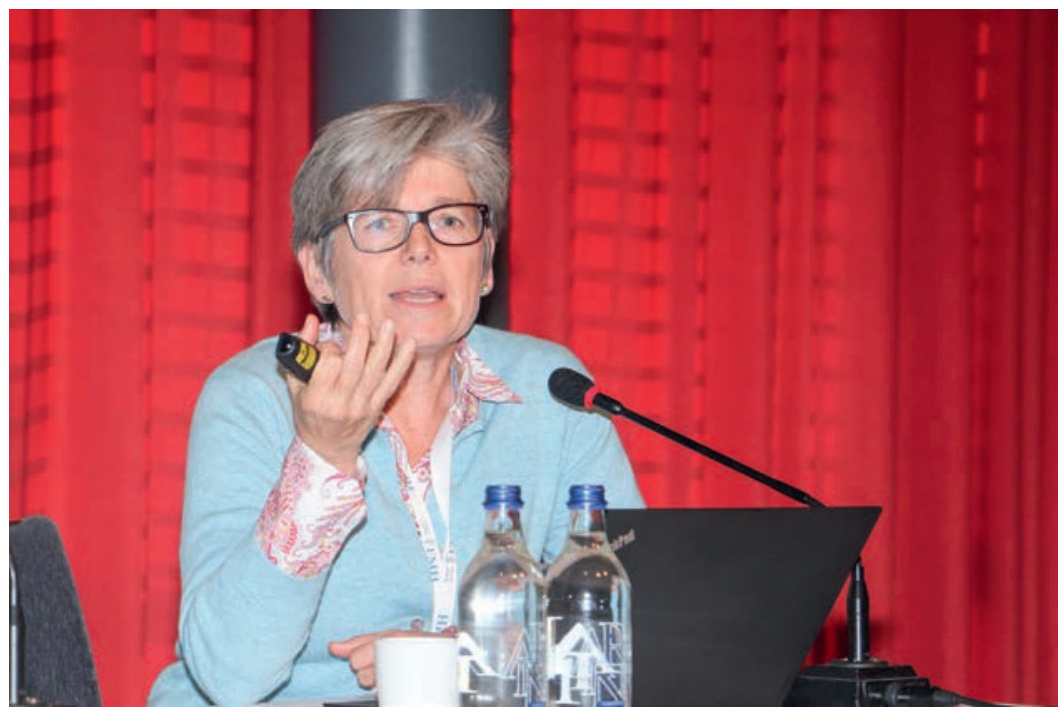

Regula Schmid dirige le groupe de travail de I'ISFM consacré aux Entrustable Professional Activities (EPA).
Kapur ont déjà été partiellement intégrées dans le Bachelor en médecine de l'EPFZ. Toutefois, son exposé portait sur la collaboration entre l'EPFZ et les sociétés de discipline médicale dans le domaine de la formation médicale postgraduée. L'EPFZ souhaite notamment créer des offres destinées aux médecins en formation postgraduée qui s'intéressent à la recherche. Un premier projet en radiologie sera lancé en 2019. Les sociétés de discipline médicale qui souhaitent évaluer une éventuelle collaboration peuvent s'adresser directement au professeur Goldhahn.

\section{Accréditation et interfaces}

Un important thème de l'année 2018 a été l'accréditation des cursus de formation postgraduée par l'OFSP. Cette accréditation s'est achevée avec succès et, comme l'a souligné Werner Bauer, le conseiller fédéral Alain Berset a certifié du haut niveau de la formation médicale postgraduée en Suisse sur la base des rapports établis par des experts suisses et étrangers. Quelques sociétés de discipline médicale ont reçu de l'administration des consignes qu'il convient désormais d'examiner et de mettre en œuvre. Dans d'autres cas, des recommandations ont été formulées. A ce sujet, l'ISFM devrait recevoir les rapports des sociétés concernées d'ici le printemps.

La bonne collaboration avec les sociétés de discipline médicale est primordiale pour l'ISFM. Werner Bauer et Christoph Hänggeli ont insisté ici sur la fonction d'interface que remplissent la Commission des titres et la Commission des établissements de formation postgraduée. Les délégués sont désignés par les sociétés de discipline médicale, mais collaborent au sein des organes de l'ISFM, remplissant ainsi un mandat étatique. La collaboration avec l'ISFM est globalement excellente, même si on constate parfois des différences considérables en termes d'efficacité et de communication. Christoph Hänggeli a rappelé que les membres des commissions pouvaient solliciter à tout moment le soutien juridique du Secrétariat général, en cas de besoin.

\section{Entrustable Professional Activities (EPA): un instrument pour les sociétés de discipline médicale?}

La notion d'Entrustable Professional Activities (EPA) définit les compétences professionnelles dont l'excellente maîtrise permet aux personnes concernées de les exercer sans supervision. Grâce aux EPA, la formation prégraduée et postgraduée, actuellement basée sur les connaissances, évoluera vers la transmission de compétences. Une tendance que l'on constate au plan inter- 
national. Dans les pays comme les Etats-Unis, les PaysBas et la Grande-Bretagne, les EPA sont déjà intégrées dans la formation postgraduée. En Suisse, les EPA font dorénavant partie intégrante de la formation médicale prégraduée.

L'ISFM a constitué un groupe de travail consacré aux EPA, qui est dirigé par la vice-présidente Regula Schmid. Ce groupe est chargé d'élaborer des directives et recommandations à l'intention des sociétés de discipline médicale et de rédiger une prise de position sur le sujet. L'important, selon Regula Schmid, est que les EPA fassent partie intégrante des objectifs de formation tout en étant intégrées dans un concept générique.

\section{Formation continue entre responsabilité personnelle et obligation}

En ce qui concerne le contrôle et la documentation de la formation continue, l'administration exerce ces derniers temps une certaine pression. La nécessité d'une réglementation renforcée de la formation continue, voire de la recertification des titres de spécialiste, a ainsi été discutée dans le cadre de la plate-forme «Avenir de la formation médicale». Selon Werner Bauer,
l'ISFM ne voit pas l'utilité de nouvelles mesures régulatrices et cette position a clairement été exprimée à la séance de la plate-forme en septembre 2018. Pour l'ISFM, l'aménagement et l'organisation de la formation continue doivent absolument rester sous la responsabilité du corps médical. Mais cette liberté est assortie d'obligations que les médecins doivent prendre au sérieux. La position de l'ISFM en la matière est consignée dans une prise de position [2] disponible sur son site Internet.

Garder la formation postgraduée et continue dans les mains du corps médical: pas besoin d'être prophète pour prévoir que cette thématique restera un sujet majeur de préoccupation pour l'ISFM et les sociétés de discipline médicale.

\section{Crédits photo}

Photos: Bruno Kesseli

\section{Références}

1 Le médecin canadien Sir William Osler (1849-1919) est souvent désigné comme le "père de la médecine moderne» en raison de son influence considérable sur la formation médicale.

2 Dans quelle direction la formation continue évolue-t-elle? Une prise de position de l'Institut suisse pour la formation médicale postgraduée et continue (ISFM). Octobre 2018. www.fmh.ch/files/ pdf21/Fortbildung_POS_PAP_Oktober_2018.pdf 